\title{
Generation of amplitude-squeezed light from a room-temperature Fabry-Perot semiconductor laser
}

\author{
J. Kitching, ${ }^{*}$ D. Provenzano, and A. Yariv \\ Department of Applied Physics, California Institute of Technology, 128-95, Pasadena, California 91125
}

Received July 3, 1995

\begin{abstract}
Amplitude-squeezed light with intensity fluctuations $29 \%$ below the standard quantum limit (SQL) is produced from a pump-suppressed room-temperature semiconductor laser, corresponding to $41 \%$ below the SQL after correction for detection efficiency. Excess noise, which degrades the observed squeezing, appears to be associated with the presence of weak longitudinal side modes.
\end{abstract}

PACS numbers: 42.50.Dv, 42.50.Lc, 42.55.Px, 42.62.Eh. (c) 1995 Optical Society of America

Squeezed states of the electromagnetic field are beginning to create new possibilities for precision measurements near the standard quantum limit (SQL). Amplitude-squeezed states generated from pump-suppressed semiconductor lasers offer a number of desirable features such as large squeezing bandwidths, ease of implementation, and the fact that the squeezing is superimposed upon a large coherent optical field. Although measurements of amplitude squeezing performed on lasers cooled to cryogenic temperatures have resulted in substantial amounts of squeezing, ${ }^{1}$ the generation of amplitude-squeezed light from room-temperature devices has been considerably less successful. To our knowledge, the largest amount of squeezing that has been measured from an unmodified semiconductor laser at room temperature was only $7 \%$ below the SQL. ${ }^{2}$ Several other experiments on both room-temperature and cryogenically cooled devices ${ }^{3-7}$ have reported substantial amounts of excess noise, which tends to obscure the squeezing.

As there appear to be no fundamental reasons why room-temperature lasers should exhibit significantly less squeezing than cryogenically cooled devices, there has been some effort recently to determine why the generation of amplitude squeezing at room temperature has been so difficult. This has been motivated in part by a desire to understand the excess noise sources that occur in semiconductor lasers. More important, however, is the lure of being able to produce large amounts of squeezing from off-the-shelf commercial devices. Several experiments were performed recently that were designed to increase the squeezing from commercial room-temperature lasers by the use of external optical modifications such as injection locking and optical feedback. ${ }^{6,7}$ Although these experiments have been, on the whole, successful in reducing the amplitude noise to as much as $50 \%$ below the SQL, the added complexity is, in principle, unnecessary.

In this Letter we present measurements, using a balanced detector, of amplitude squeezing 29\% below the SQL from a room-temperature semiconductor laser. This corresponds to a noise level $41 \%$ below the SQL at the output facet of the laser when correction is made for optical losses present in the detection system. These results demonstrate that substantial squeezing can indeed be obtained from a commercial device operating at room temperature with no external components or modifications other than those used to detect the light. The excess noise that degrades the squeezing is also investigated, and it appears that the source of most of this noise under a wide range of operating conditions is associated with the presence of longitudinal side modes. Our measurements indicate that, under optimal conditions, the amount of squeezing is limited primarily by the device and detection efficiencies, in accordance with the standard singlemode theory of amplitude noise in semiconductor lasers. ${ }^{8,9}$

The experimental setup is shown in Fig. 1. The semiconductor laser used (SDL-5402-H1) was an actively temperature-stabilized quantum-well indexguided structure with an external differential quantum efficiency of $68 \%$ at room temperature. The output light from the laser was sent through an optical isolator (providing $>60 \mathrm{~dB}$ of isolation) to a delay line balanced receiver ${ }^{3}$ for measurement of the amplitude noise. Common mode rejection of more than $45 \mathrm{~dB}$ was obtained at a frequency of $28 \mathrm{MHz}$. Figure 2 shows the measured photocurrent noise power spectrum (trace A) when the photocurrents at $28 \mathrm{MHz}$ were added. The electronic delay present in one arm of the receiver caused a frequency-dependent phase shift between the two signals entering the differential amplifier with the result that the output photo-

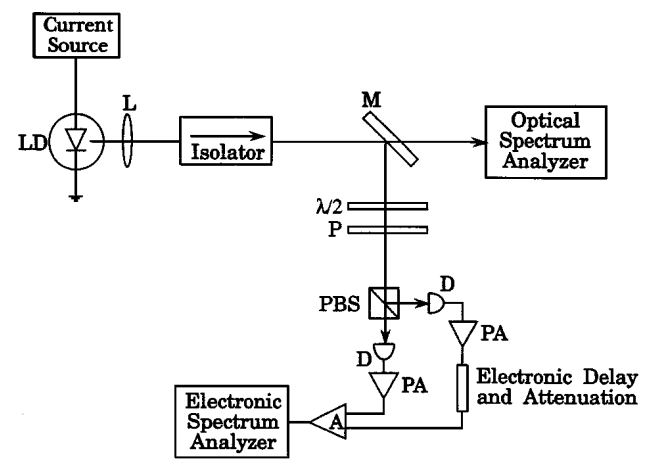

Fig. 1. Experimental setup: LD, laser diode; L, lens; M, mirror; $\lambda / 2$, half-wave plate; $\mathrm{P}$, polarizer; PBS, polarizing beam splitter; D's, detectors (Hamamatsu S3994); PA's, preamplifiers (Minicircuits ZFL-500LN); A, differential amplifier (Tektronix 7A24). 
current noise power from the receiver varied, as a function of frequency, between the actual laser noise level and the SQL. ${ }^{3}$ We checked the level of the SQL by shining the light from high-power light-emitting diodes (LED's) onto the detectors to produce the same photocurrent as did the laser. The photocurrent noise power spectrum obtained from the LED's is shown in trace B. It can be seen that, at $28 \mathrm{MHz}$, the laser photocurrent noise power is $1.4 \mathrm{~dB}$ below the SQL (assumed, here, to be determined by the LED), indicating that the laser amplitude fluctuations were squeezed by this amount. Whereas LED's have also been shown to exhibit squeezed intensity fluctuations,${ }^{10}$ the low efficiency of the devices $(<10 \%$, current to current) and the close agreement of the LED noise level with the SQL measured with the laser noise spectrum (see Fig. 2, trace A, at 7 and $20 \mathrm{MHz}$ ) indicated that the LED noise was indeed close to the SQL. For the measurement in Fig. 2 the laser injection current was $66 \mathrm{~mA}$, the current-to-current differential efficiency was $48 \%$, and the laser was cooled to $\sim 5{ }^{\circ} \mathrm{C}$. The resolution bandwidth for this measurement was $100 \mathrm{kHz}$, and the background amplifier noise level (typically $>10 \mathrm{~dB}$ below the signal level for the higher detector photocurrents) was subtracted from all measured signals.

Saturation of the detector response at high frequencies when the detector is illuminated with intense optical fields can result in errors in the measurement of the laser noise or in the calibration of the SQL. However, previous experiments ${ }^{6,7}$ with the detectors used in this experiment demonstrated their excellent saturation properties, and no evidence for significant detector saturation was observed in this experiment within the range of photocurrents and frequencies at which measurements were made. At detector currents up to $14 \mathrm{~mA}$ the shot-noise level at $28 \mathrm{MHz}$, calibrated with both the LED's and the laser, was found to be linear in the dc photocurrent to within roughly $1 \%$. As an additional check on the level of squeezing, the amplitude noise was measured as a function of the optical attenuation. ${ }^{7}$ As expected, the noise power, normalized to the SQL, was found to increase in a linear fashion toward unity as the attenuation was made stronger.

The squeezing could be obtained only within a narrow range of laser temperatures and injection currents; at most combinations of these two parameters the laser operated multimode and the amplitude noise was far above the SQL. It appeared that particularly good side-mode suppression was required in order generate squeezed light from the laser. The longitudinal mode spectrum was therefore measured simultaneously with the amplitude noise, and a typical spectrum, obtained when the amplitude noise was $1.4 \mathrm{~dB}$ below the SQL, is shown in Fig. 3. It seems likely that the excellent side-mode suppression $(\approx 30 \mathrm{~dB})$ observed in this laser was the reason for the large squeezing measured, whereas other, similar, lasers would not produce an equivalent amount of amplitude squeezing under comparable operating conditions. ${ }^{6,7,11}$

The photocurrent noise at $28 \mathrm{MHz}$ was then measured as a function of the laser injection current. The results, normalized to the SQL, are plotted in Fig. 4 against the pump rate $R=i / i_{\text {th }}-1$. Also shown in the figure is the prediction of the single-mode theor $y^{9}$ in the low-frequency limit with the pump noise assumed to be completely suppressed. The minimum noise obtained with this laser was $1.5 \mathrm{~dB}$, or $29 \%$ below the SQL.

If the laser temperature was altered slightly, both the mode spectrum and the amplitude noise were found to change in a correlated way. By far the largest change in the side-mode power occurred for the two longitudinal side modes closest to the lasing mode in wavelength. A clear correlation between the optical power in these two side modes and excess amplitude noise can be seen in Fig. 5; the laser noise was found to increase to ten times the

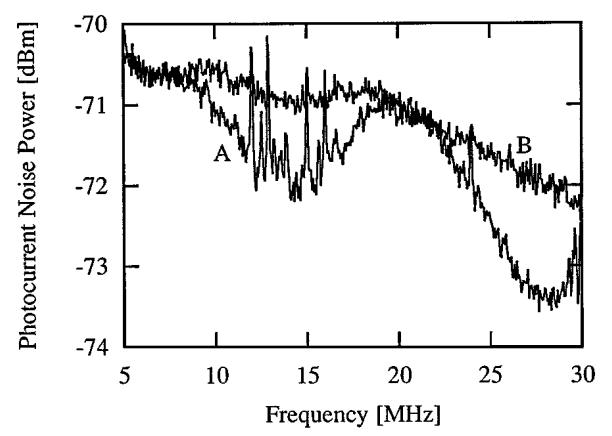

Fig. 2. Photocurrent noise power spectrum from the laser (trace A) and the LED (trace B) when the differential amplifier added the detector photocurrents at $28 \mathrm{MHz}$. Squeezing is indicated by the laser noise dropping below the LED noise (SQL) at certain frequencies.

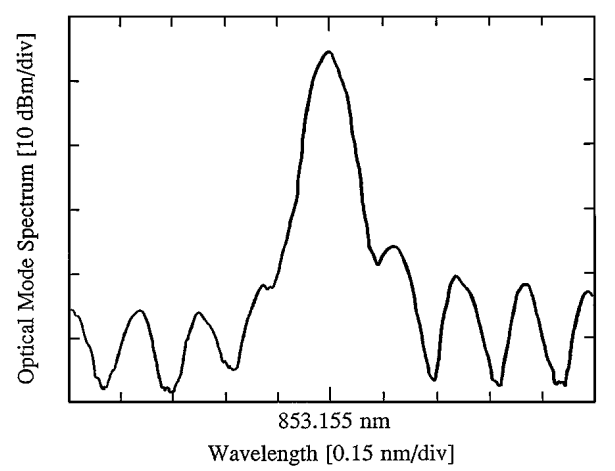

Fig. 3. Longitudinal mode spectrum for the laser when the amplitude noise was squeezed by $1.4 \mathrm{~dB}$.

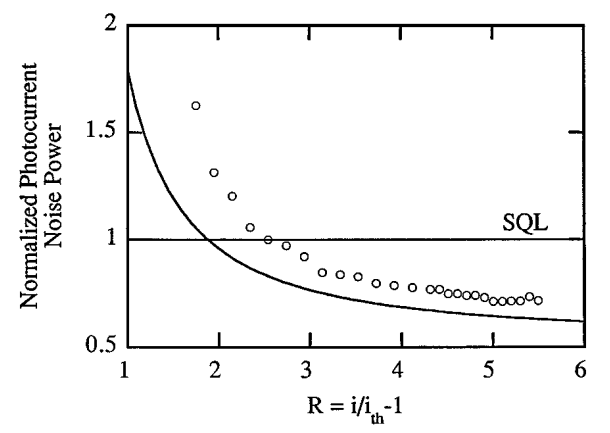

Fig. 4. Normalized photocurrent noise power spectrum dependence on injection current. The solid curve is the prediction of the single-mode theory of Ref. 9. 


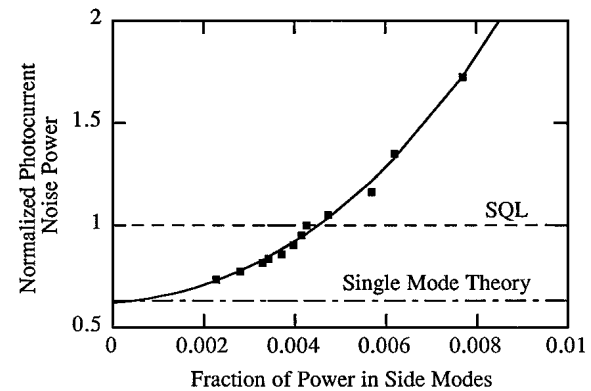

Fig. 5. Dependence of the laser amplitude noise on the fraction of power in the two side modes closest to the lasing mode. The solid curve is a third-order polynomial fit to the measured data.

SQL when 5\% of the optical power was present in the two measured side modes. An extrapolation of a third-order polynomial fit (solid curve) to zero side-mode power gives excellent agreement with the prediction of the single-mode theory (indicated by the dashed-dotted line). This suggests that if the excess noise associated with the side modes were eliminated the laser noise would approach the fundamental limit determined, primarily, by the device and detection efficiencies. We note, however, that the theory of Ref. 9 applies only to a laser for which both facet reflectivities are close to unity. This was not the case for the laser used in this experiment, which had one facet antireflection coated. Recent numerical calculations using traveling-wave models ${ }^{12}$ have shown that dramatic changes in the theoretically predicted amplitude noise power can occur when the good-cavity assumption is relaxed.

The mechanism by which the side modes generate the excess noise is still unclear. However, the secondorder term in the fit dominates over the range of side-mode powers shown, indicating that the excess noise is proportional to the square of the side-mode power in this range. Attempts to fit the multimode theory described in a previous publication ${ }^{7}$ to the data were not particularly successful. At side-mode powers above $1 \%$ of the total power the fit in Fig. 5 no longer agreed well with the measured data.

It is known that an extremely small amount of optical feedback can significantly affect the amplitude noise of the laser. ${ }^{7}$ We therefore performed several checks under conditions of maximum squeezing to determine whether spurious optical feedback was present and, if so, was influencing the laser noise. First, the laser temperature and the injection current were varied by an amount not large enough to cause a significant change in the side-mode suppression but large enough to vary the laser frequency by a few gigahertz. The idea here was that if optical feedback were present with an intensity large enough to change the amplitude noise, then changing the laser frequency would change the phase of the feedback, thereby generating oscillations in the amplitude noise. The amplitude noise was found to vary by less than the measurement error of $0.1 \mathrm{~dB}$, however. In addition, we varied the positions of both the optical isolator (which was found to be the offending component in a previous experiment ${ }^{7}$ ) and the collimating lens over several wavelengths by placing their translation stages on piezoelectric transducers, and again no significant change in the amplitude noise was observed. The above checks seem to indicate that optical feedback from the tested components was not playing a significant role in reducing the amplitude noise.

In summary, amplitude squeezing $29 \%$ below the SQL has been measured from a commercial room-temperature Fabry-Perot semiconductor laser. Excess noise, which degrades the squeezing, appears to be associated with the presence of longitudinal side modes. Although the measured amount of squeezing is still $10-20 \%$ smaller than predicted by the standard single-mode theory, an extrapolation of the data to zero side-mode power gives agreement with the theory to within $\approx 3 \%$. Careful stabilization of the laser temperature and injection current are important factors in keeping the side-mode power to below acceptable levels. However, other than the control of optical feedback from the detection system, no other modifications to the laser appear to be necessary to yield significant amplitude squeezing.

The authors gratefully acknowledge useful comments from Y. Shevy and support from the National Science Foundation, the Advanced Research Projects Agency, and the U.S. Office of Naval Research. The laser used in the experiment was generously donated by Spectra Diode Laboratories, Inc.

*Present address, National Institute of Standards and Technology, M.S. 847.10, 325 Broadway, Boulder, Colorado 80303.

\section{References}

1. W. H. Richardson, S. Machida, and Y. Yamamoto, Phys. Rev. Lett. 66, 2867 (1991).

2. S. Machida, Y. Yamamoto, and Y. Itaya, Phys. Rev. Lett. 58, 1000 (1987).

3. G. L. Abbas, V. W. S. Chan, and T. K. Yee, Opt. Lett. 8, 419 (1983); S. Machida and Y. Yamamoto, Opt. Lett. 14, 1045 (1989).

4. W. H. Richardson and R. M. Shelby, Phys. Rev. Lett. 64, 400 (1990).

5. M. J. Freeman, H. Wang, D. G. Steel, R. Craig, and D. R. Scifres, Opt. Lett. 18, 379 (1993).

6. H. Wang, M. J. Freeman, and D. G. Steel, Phys. Rev. Lett. 71, 3951 (1993); M. J. Freeman, H. Wang, D. G. Steel, R. Craig, and D. R. Scifres, Opt. Lett. 18, 2141 (1993).

7. J. Kitching, A. Yariv, and Y. Shevy, Phys. Rev. Lett. 74, 3371 (1995).

8. D. E. McCumber, Phys. Rev. 141, 306 (1966); H. Haug and H. Haken, Z. Phys. 204, 262 (1967).

9. Y. Yamamoto, S. Machida, and O. Nilsson, Phys. Rev. A 34, 4025 (1986); 35, 5114 (1987).

10. P. R. Tapster, J. G. Rarity, and J. S. Satchell, Europhys. Lett. 4, 293 (1987); H.-A. Bachor, P. Rottengatter, and C. M. Savage, Appl. Phys. B 55, 258 (1992).

11. M. J. Freeman, D. C. Kilper, D. G. Steel, R. Craig, and D. R. Scifres, Opt. Lett. 20, 183 (1995).

12. B. Tromberg, H. E. Lassen, and H. Olesen, IEEE J. Quantum Electron. 30, 939 (1994); D. D. Marcenac and J. E. Carroll, IEEE J. Quantum Electron. 30, 2064 (1994). 